\title{
Éditorial
}

\section{La soumission chimique}

$\mathrm{L}$

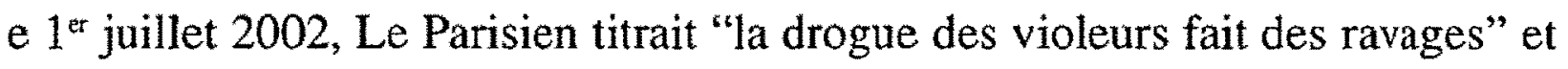
continuait par "on a volé aux victimes un moment de leur vie".

La traque du GHB ne faisait que commencer ...

La prévalence des crimes liés à la soumission chimique est difficile à évaluer. La presse se fait souvent l'écho d'agressions qui commencent dans un cadre festif par l'introduction de substances dans les verres, mais les statistiques françaises sont particulièrement pauvres. Le GHB est-il à la mode, comme l'était, à une époque, le flunitrazépam, avant que sa présentation galénique ne soit modifiée?

La quasi-impossibilité à mettre en évidence, dans les cas de violences sexuelles, le GHB contribue certainement à positionner ce produit comme un mythe. Cette difficulté n'est en rien analytique (le contrôle de qualité récemment organisé en est l'illustration), mais fonction d'une très rapide élimination plasmatique et urinaire. Si l'on ajoute à cela les déclarations tardives des victimes, l'effet amnésiant de la plupart des agents psycho-actifs utilisés, les faibles concentrations circulantes (qui nécessitent l'emploi de techniques séparatives obligatoirement couplées à la spectrométrie de masse - la MS/MS étant l'approche de choix) ou encore l'absence de cheveux pour documenter l'expertise, il apparaît que la soumission chimique est répandue dans toute la société.

Sans vouloir être alarmiste, il nous a semblé important que la SFTA se penche sur ce problème, afin de proposer un numéro spécial sur la soumission chimique, recueil de référence ou de synthèse sur le sujet. C'est pourquoi, les colonnes du Journal ont également été ouvertes à des cliniciens, en prolongement du congrès de la Société de Toxicologie Clinique à Djerba, en mars 2002.

Plus que jamais, il me semble indispensable, dans ce domaine si particulier, que les toxicologues travaillent en réseau, d'une part en confrontant leurs analyses avec les observations médicales, et d'autre part en servant de relais pour transférer une partie des échantillons vers des laboratoires pratiquant en routine ces investigations. $\mathrm{La}$ prise en charge des victimes passe aussi par une analyse toxicologique poussée à l'extrême.

L'aspect multi-facettes de la soumission chimique me semble avoir été abordé dans ce numéro spécial par un panel de professionnels de l'expertise judiciaire. Qu'ils soient tous remerciés d'avoir accepté de partager leur expérience.

Pascal KINTZ. 